\title{
ChemComm
}

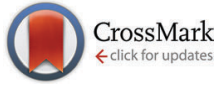

Cite this: Chem. Commun., 2015, 51,8861

Received 5th March 2015, Accepted 16th April 2015

DOI: $10.1039 / c 5 c c 01812 a$

www.rsc.org/chemcomm

\section{Photoreactivity of the linker region of two consecutive G-quadruplexes formed by human telomeric DNA $\dagger$}

\author{
Yue Li $i^{\mathrm{a}}$ and Hiroshi Sugiyama*ab
}

\begin{abstract}
We report the application of a photoreaction method for probing two consecutive G-quadruplexes formed by human telomeric DNA. This method can discriminate the loop structure located between two consecutive G-quadruplexes formed by eight TTAGGG repeats in $\mathrm{K}^{+}$and $\mathrm{Na}^{+}$solutions.
\end{abstract}

The human telomeric DNA contains thousands of tandem TTAGGG repeats and an approximately 200 bp $3^{\prime}$ singlestranded overhang that is capable of forming G-quadruplex structures. ${ }^{1}$ Recent visualization studies provided evidence of G-quadruplex formation at the telomeric region in cells. ${ }^{2}$ Stable G-quadruplexes formed by telomere overhang can protect chromosomes from degradation, end fusion, and being recognized as double-strand breaks (DSBs). ${ }^{3,4}$ To date, various intramolecular G-quadruplex structures formed by four TTAGGG repeats in the presence of $\mathrm{K}^{+}$or $\mathrm{Na}^{+}$ions have been elucidated. ${ }^{5-9}$ However, this view of single G-quadruplex formation is incomplete, as higher-order G-quadruplex structures are more biologically relevant considering the existence of the long telomere repeats. The consecutive G-quadruplexes formed by longer telomeric DNA have been investigated in several experimental studies, including those that used CD spectra, NMR, atomic force microscopy (AFM), and optical tweezers. ${ }^{10-14}$ Moreover, the study of higherorder G-quadruplex structures offers new and selective binding sites for drugs that target telomeric sequences. Several ligands that can selectively bind to higher-order G-quadruplexes have been reported. ${ }^{15,16}$

Because the single G-quadruplex units of the telomeric sequence in $\mathrm{K}^{+}$and $\mathrm{Na}^{+}$solutions are highly polymorphic, various higher-order G-quadruplex structures may be formed by long telomeric DNA. ${ }^{17}$ The sequence of eight TTAGGG repeats

\footnotetext{
${ }^{a}$ Department of Chemistry, Graduate School of Science, Kyoto University,

Kitashirakawa-oiwakecho, Sakyo-ku, Kyoto 606-8502, Japan.

E-mail: hs@kuchem.kyoto-u.ac.jp

${ }^{b}$ Institute for Integrated Cell-Material Sciences (WPI-iCeMS), Kyoto University,

Yoshida-ushinomiyacho, Sakyo-ku, Kyoto 606-8501, Japan

$\dagger$ Electronic supplementary information (ESI) available. See DOI: 10.1039/ c5cc01812a
}

and its variant sequences have been frequently used in the study of higher-order structures. It was suggested that these sequences could form two consecutive G-quadruplexes that were connected by a TTA loop. ${ }^{18,19}$ Stable consecutive G-quadruplex structures can be formed by two hybrid G-quadruplexes (hybrid-1 or hybrid-2) in $\mathrm{K}^{+}$solution, or by two antiparallel G-quadruplexes in $\mathrm{Na}^{+}$ solution. Molecular modelling studies indicate that the stacking model of hybrid-1 and hybrid- 2 was most stable in $\mathrm{K}^{+}$solution. $^{20}$ As single G-quadruplex formation is quite different in $\mathrm{K}^{+}$and $\mathrm{Na}^{+}$ solutions, consecutive G-quadruplex structures formed by two G-quadruplex subunits have different stacking interactions and TTA loop structures in the linker region.

Conversely, the thymine (T) base of DNA can be replaced with 5-halouracil $\left({ }^{\mathrm{Br}} \mathrm{U}\right.$ or $\left.{ }^{\mathrm{I}} \mathrm{U}\right)$. We have demonstrated that the photoreactions of 5-halouracil-containing DNA can be used to probe various conformations of DNA, including the A, B, and $\mathrm{Z}$ forms and G-quadruplexes. ${ }^{21-25}$ Under $302 \mathrm{~nm}$ irradiation, the photochemical products formed by hydrogen abstraction are highly dependent on conformation. Regarding G-quadruplexes, the photoreaction efficiency and products are closely related to the loop structure. In our previous study, we found that the 2 -deoxyribonolactone residue was effectively and selectively produced in the diagonal loop of the antiparallel G-quadruplexes formed by 5-iodouracil-containing telomeric DNA. However, there was almost no photoreactivity in the loop structures of hybrid G-quadruplexes formed in $\mathrm{K}^{+}$solution. ${ }^{26}$

In this study, we applied our photoreaction method to detect the loop structure located between two consecutive G-quadruplexes formed by eight TTAGGG repeats in $\mathrm{K}^{+}$and $\mathrm{Na}^{+}$solutions. The second thymine of the TTA loop was replaced with ${ }^{\mathrm{Br}} \mathrm{U}$, as it was most reactive in the diagonal loop of an antiparallel G-quadruplex in the case of ${ }^{\mathrm{I}} \mathrm{U}$. We proved that, during the photoreaction process, the cleavage of $\mathrm{C}-\mathrm{Br}$ occurs via a heterolysis pathway, whereas the cleavage of $\mathrm{C}-\mathrm{I}$ occurs via both homolysis and heterolysis pathways. ${ }^{27}$ It was also reported that electron transfer can occur from a G-quadruplex to the $\mathrm{T}=\mathrm{T}$ dimer, which is a chemical electron trap, similar to ${ }^{\mathrm{Br}} \mathrm{U}^{28}$ Therefore, we used ${ }^{\mathrm{Br}} \mathrm{U}$ instead of ${ }^{\mathrm{I}} \mathrm{U}$ to focus on the electron transfer from G-tetrads to ${ }^{\mathrm{Br}} \mathrm{U}$ during the photoreaction process. 
Table 1 Oligonucleotides used in this study

\begin{tabular}{|c|c|}
\hline Oligonucleotides & Sequence $\left(5^{\prime}\right.$ to $\left.3^{\prime}\right)$ \\
\hline ODN 1 & FAM-TTAGGGTTAGGGT ${ }^{\mathrm{Br}}$ UAGGGTTAGGG \\
\hline ODN 2 & 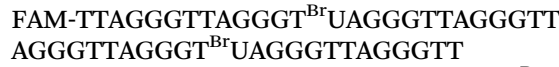 \\
\hline ODN 3 & $\begin{array}{l}\text { FAM-TTAGGGTTAGGGTTAGGGTTAGGGT } \\
\text { UAGGGTTAGGGTTAGGGTTAGGGTT }\end{array}$ \\
\hline ODN 4 & FAM-TTAGGGTTAGGG \\
\hline ODN 5 & FAM-TTAGGGTTAGGGTTAGGGTTAGGG \\
\hline ODN 6 & $\begin{array}{l}\text { FAM-TTAGGGTTAGGGTTAGGGTTAGGGTT } \\
\text { AGGGTTAGGG }\end{array}$ \\
\hline
\end{tabular}

All of the oligonucleotides used in this study are shown in Table 1. ODN 1, with four TTAGGG repeats, was designed to confirm the photoreaction in the diagonal loop of an antiparallel G-quadruplex reported previously. ODN 2, which may form two consecutive antiparallel G-quadruplexes, had two ${ }^{\mathrm{Br}} \mathrm{U}$ replacements in the diagonal loops. It was designed to study the photoreaction of the diagonal loop in two consecutive G-quadruplexes formed in $\mathrm{Na}^{+}$solution. ODN 3, with a ${ }^{\mathrm{Br}} \mathrm{U}$ replacement in the TTA loop located between two G-quadruplex-forming sequences, was designed to investigate the photoreaction of the loop located between G-quadruplexes in $\mathrm{Na}^{+}$and $\mathrm{K}^{+}$solutions. ODN 4-6 were used as reference DNAs. All ODNs were $5^{\prime}$-end FAM labeled.

The CD spectra were analyzed to confirm the G-quadruplex formation. As shown in Fig. S1 (ESI $\dagger$ ), both ODN 1 and ODN 2 formed antiparallel G-quadruplexes in $\mathrm{Na}^{+}$solution and hybrid G-quadruplexes in $\mathrm{K}^{+}$solution. The CD signals of ODN 2, which contained eight TTAGGG repeats, in $\mathrm{Na}^{+}$and $\mathrm{K}^{+}$solution had about two times the amplitude of the CD signals of ODN 1 in $\mathrm{Na}^{+}$and $\mathrm{K}^{+}$solutions, respectively. Therefore, the eight TTAGGG repeats labeled with FAM at the $5^{\prime}$ end used in the current study might mainly form two consecutive G-quadruplex structures. ${ }^{20}$

Previously, we demonstrated a proposed mechanism in the hot-spot sequence $\left(5^{\prime}-(\mathrm{G} / \mathrm{C})[\mathrm{A}]_{n=1,2}{ }^{\mathrm{Br}} \mathrm{U}^{\mathrm{Br}} \mathrm{U}-3^{\prime}\right)$ of duplex DNA and its reverse sequence (Fig. S2, ESI $\dagger$ ). ${ }^{23,24}$ Similar to duplex DNA, 2 '-deoxyribonolactone products were obtained from the photoreaction of G-quadruplexes in our previous study. ${ }^{26}$ The formation of guanine radical cations in G-quadruplexes was investigated using transient UV-vis spectra and time-dependent DFT study. ${ }^{29,30}$ Therefore, we assumed that a similar electron transfer mechanism occurs in the photoreaction of G-quadruplexes. Based on this mechanism, DNA samples in different ion solutions were photoirradiated separately at $0{ }^{\circ} \mathrm{C}$ for 15 min using a monochromator $(302 \mathrm{~nm})$, and were then subjected to heating and cleavage at $95{ }^{\circ} \mathrm{C}$ for $15 \mathrm{~min}$. Subsequently, the resulting $5^{\prime}$-end FAM-labeled DNA fragments were analyzed by denaturing gel analysis. Finally, photoreactive sites were determined by comparison with the reference ODNs.

The results obtained for ODN 1 are shown in Fig. 1a and Fig. S3 (ESI $\dagger$ ). In the presence of $\mathrm{Na}^{+}$, efficient cleavage was observed after heating, indicating the formation of deoxyribonolactone products in the diagonal loop of the antiparallel G-quadruplex. The product of the photoreaction also showed a similar migration in the gel $v s$. the reference DNA ODN 4. However, in $\mathrm{K}^{+}$and $\mathrm{Li}^{+}$solutions, we did not observe clear bands of deoxyribonolactone photoreaction products. These results were (a)
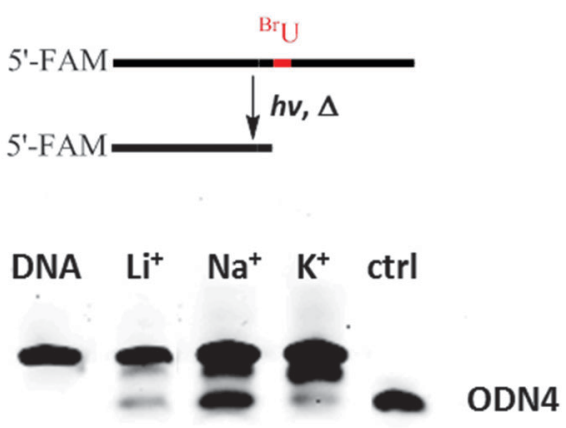

(b)
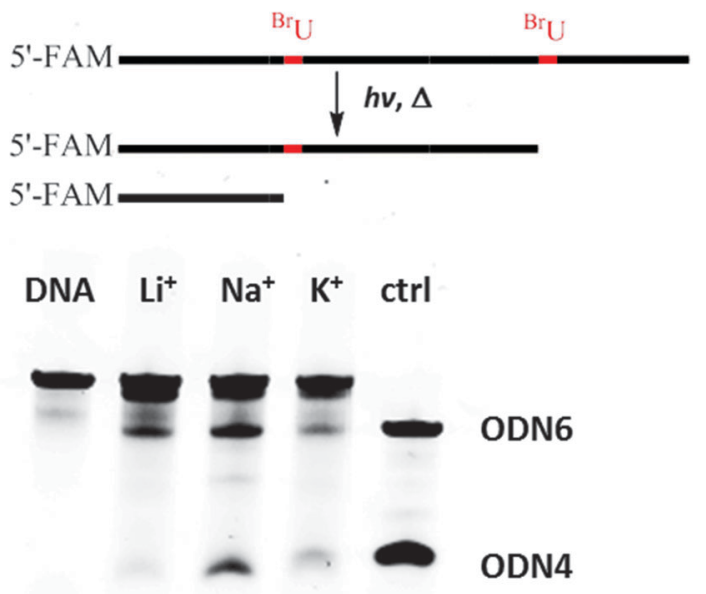

Fig. 1 Photoreaction of ODN 1 (a) and ODN 2 (b) in $\mathrm{Li}^{+}$solution $(20 \mathrm{mM}$ lithium cacodylate, $\mathrm{pH} 7.0,80 \mathrm{mM} \mathrm{LiCl}), \mathrm{Na}^{+}$solution $(20 \mathrm{mM}$ lithium cacodylate, $\mathrm{pH} 7.0,100 \mathrm{mM} \mathrm{NaCl}$ ), and $\mathrm{K}^{+}$solution ( $20 \mathrm{mM}$ lithium cacodylate, $\mathrm{pH}$ 7.0, $100 \mathrm{mM} \mathrm{KCl).} \mathrm{ODN} 4$ and ODN 6 were used as reference DNAs.

in accordance with the photoreaction of 5-iodouracil-containing telomeric DNA revealed in a previous HPLC analysis. ${ }^{26}$ Therefore, the deoxyribonolactone products of photoreactions and photoreaction efficiency can be investigated via gel analysis using $5^{\prime}$-end FAM-labeled DNA. We also calculated the efficiency of photoreaction by measuring the percentage of the cleavage band. For ODN 1, the efficiency of the photoreaction using 15 min of irradiation was about $35 \%$.

Next, we investigated the photoreaction of ODN 2 and the results were shown in Fig. 1b. ODN 4 and ODN 6 were used as reference DNAs for gel analysis. Two cleavage bands that showed similar migration to those of ODN 4 and ODN 6 were obtained for the products of ODN 2 in $\mathrm{Na}^{+}$solution. Therefore, ODN 2 showed efficient photoreaction in two diagonal loops in the presence of $\mathrm{Na}^{+}$, suggesting the formation of two antiparallel G-quadruplexes by ODN 2. These two bands showed different intensity, suggesting the two diagonal loop structures were not exactly the same. In contrast, gel analysis showed that no efficient photoreaction occurred in the presence of $\mathrm{K}^{+}$. One band with similar gel migration to that of ODN 6 was observed in $\mathrm{Li}^{+}$ solution. The CD spectra revealed that ODN 2 formed a stable hairpin structure. Therefore, we assumed that this band might be a result of the photoreaction cleavage of the hairpin structure. The results obtained for ODN 1 and ODN 2 led us to conclude that efficient photoreaction occurred in the diagonal loop of 


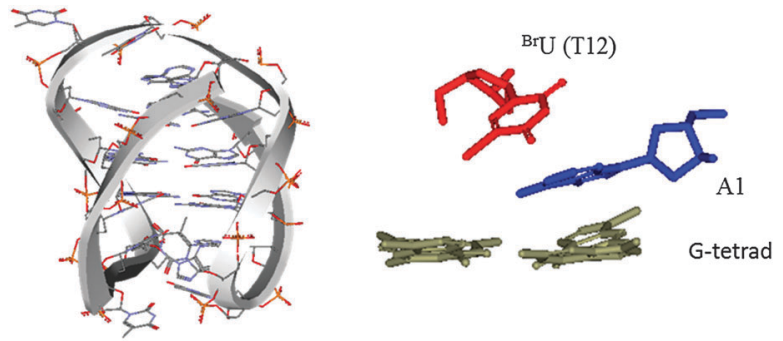

Fig. 2 NMR structure of an antiparallel G-quadruplex in $\mathrm{Na}^{+}$solution (PDB code 143D, 5'-AGGGTTAGGGTTAGGGTTAGGG-3') and a stacking model of thymine (yellow), 5-bromouracil (red), and adenine (blue) in the diagonal loop region.

antiparallel G-quadruplexes formed by human telomeric sequences. Furthermore, the two cleavage bands of ODN 2 showed that ODN 2 might form two consecutive antiparallel G-quadruplexes. In contrast, no efficient photoreaction was found in these loop structures of the hybrid G-quadruplexes formed in $\mathrm{K}^{+}$solution.

The diagonal loop structure of an antiparallel G-quadruplex in $\mathrm{Na}^{+}$solution is shown in Fig. 2. A1 was positioned between ${ }^{\mathrm{Br}} \mathrm{U}(\mathrm{T} 12)$ and the G-tetrad, and was directly stacked with ${ }^{\mathrm{Br}} \mathrm{U}(\mathrm{T} 12)$. Considering the photoreaction mechanism of hot-spot sequences in duplex DNA, we proposed that A1 prevented rapid electron back transfer, and increased the lifetime of the anion radical formed by ${ }^{\mathrm{Br}} \mathrm{U}$. Therefore, an efficient photoreaction occurred in the diagonal loop of the antiparallel G-quadruplex formed by ODN 1 in $\mathrm{Na}^{+}$solution. Moreover, ODN 2, which might form two consecutive antiparallel G-quadruplexes with two diagonal loops, exhibited two photoreaction cleavage sites. However, in the hybrid-1 and hybrid-2 G-quadruplex structures there were no such stacking models in the loop structures (Fig. S4, ESI $\dagger$ ).

Subsequently, we investigated the photoreaction of ODN 3, in which one thymine in the loop located between two G-quadruplexforming sequences was substituted with ${ }^{\mathrm{Br}} \mathrm{U}$. ODN 5, which contains four TTAGGG repeats, was the reference DNA. As shown in Fig. 3a, a clear cleavage band similar to that of ODN 5 was observed in the presence of $\mathrm{K}^{+}$. However, in $\mathrm{Na}^{+}$solution, the photoreaction efficiency was lower compared with the photoreaction efficiency observed in $\mathrm{K}^{+}$solution. To confirm the photoreaction of ODN 3 in $\mathrm{K}^{+}$, we checked the photoreaction products before and after heating and cleavage. Fig. $3 \mathrm{~b}$ clearly shows that, after $15 \mathrm{~min}$ of heating at $95{ }^{\circ} \mathrm{C}$, a cleavage band of deoxyribonolactone products appeared.

We carried out an additional photoreaction using different irradiation times $(5,10,15,20$ and $30 \mathrm{~min})$. As shown in Fig. S5 (ESI $\dagger$ ), the time-dependent photoreactions of ODN 1 in $\mathrm{Na}^{+}$, ODN 2 in $\mathrm{Na}^{+}$solution and ODN 3 in $\mathrm{K}^{+}$solution were compared. Generally, the photoreactions of ODN 1 and ODN 2 in $\mathrm{Na}^{+}$solution were more efficient than the photoreaction of ODN 3 in $\mathrm{K}^{+}$solution. The fast kinetics in $\mathrm{Na}^{+}$solution suggested that antiparallel quadruplexes could be easily formed in $\mathrm{Na}^{+}$ solution and the interaction of two antiparallel G-quadruplexes was not strong. Thus, the two consecutive G-quadruplexes in $\mathrm{Na}^{+}$ solution showed even higher photoreaction efficiency than that of single antiparallel G-quadruplex. In contrast, in the two (a)

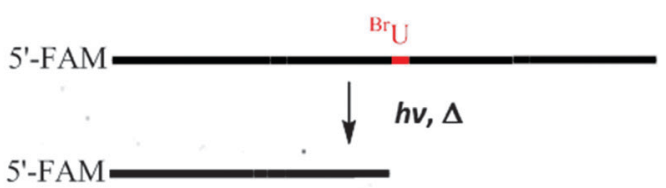

(b)
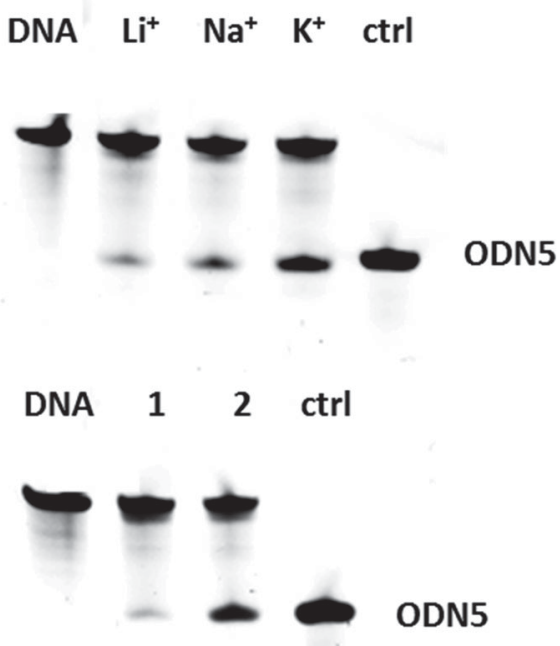

Fig. 3 (a) Photoreaction of ODN 3 in $\mathrm{Li}^{+}$solution $(20 \mathrm{mM}$ lithium cacodylate, $\mathrm{pH} 7.0,80 \mathrm{mM} \mathrm{LiCl}$ ), $\mathrm{Na}^{+}$solution ( $20 \mathrm{mM}$ lithium cacodylate, $\mathrm{pH} 7.0,100 \mathrm{mM}$ $\mathrm{NaCl}$ ), and $\mathrm{K}^{+}$solution ( $20 \mathrm{mM}$ lithium cacodylate, $\mathrm{pH} 7.0,100 \mathrm{mM} \mathrm{KCl}$ ). (b) Gel analysis of the photoreaction of ODN 3 in $\mathrm{K}^{+}$solution before (band 1) and after (band 2) heating cleavage. ODN 5 was used as the reference DNA.

consecutive structures formed by hybrid-1 and hybrid-2, the interaction was strong and the linker loop region between the two G-quadruplexes was stable. Therefore, the photoreaction kinetics of ODN 3 in $\mathrm{K}^{+}$solution was lower than that of ODN 2 in $\mathrm{Na}^{+}$solution.

To compare the TTA loop structures of two G-quadruplex subunits formed by eight TTAGGG repeats in $\mathrm{Na}^{+}$and $\mathrm{K}^{+}$ solutions, we constructed two consecutive structures according to a method reported previously, and compared their linker regions based on the results of molecular simulation. ${ }^{20}$ The dimeric model of two antiparallel units in $\mathrm{Na}^{+}$solution and the dimeric model of hybrid- 1 and hybrid- 2 in $\mathrm{K}^{+}$solution were generated as shown in Fig. 4. First, molecular simulation showed that the consecutive structure formed by hybrid-1 and hybrid-2 quadruplexes in $\mathrm{K}^{+}$solution had stronger stacking interactions, in which the TTA linker was involved. In contrast, the interaction of two antiparallel G-quadruplexes in $\mathrm{Na}^{+}$ solution was not strong and the two thymines of the TTA linker were not well stacked with the G-tetrads of the two G-quadruplexes. Second, in the TTA loop located between the hybrid-1 and hybrid-2 structures, two adenines (A19 and A37) were positioned between the stacking of thymines $\left({ }^{\mathrm{Br}} \mathrm{U}\right)$ and G-tetrads. Similar to what was observed for the hot-spot sequence in duplex DNA and the diagonal loop of the antiparallel G-quadruplex, these two adenines prevented rapid electron back transfer, and increased the lifetime of the anion radical formed by ${ }^{\mathrm{Br}} \mathrm{U}$. However, in the case of a dimeric model 
(a)

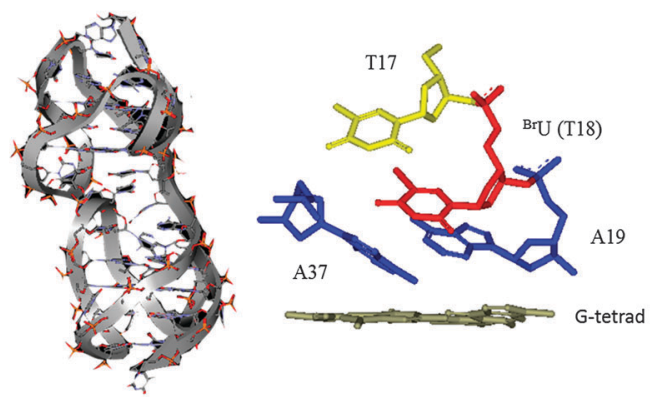

(b)
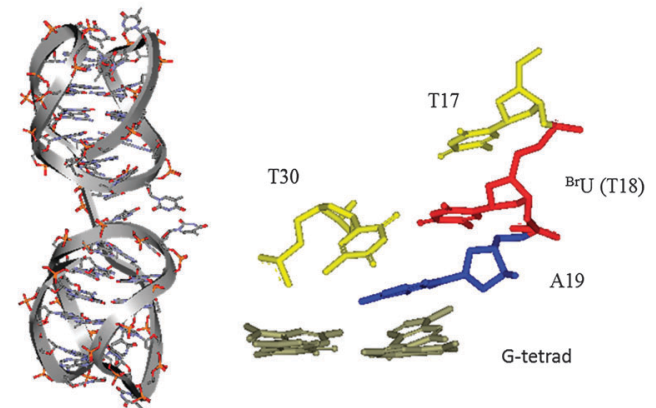

Fig. 4 The dimeric models of two consecutive G-quadruplexes in $\mathrm{K}^{+}$ (a) and $\mathrm{Na}^{+}$(b) solutions and the stacking model of thymines (yellow), 5-bromouracil (red), and adenines (blue) in the linker loop region.

in $\mathrm{Na}^{+}$solution, there was no such stacking in the TTA loop between two antiparallel G-quadruplexes. A19 stacked with T30, which was located in the diagonal loop of one antiparallel G-quadruplex. Finally, in the TTA loop located between the hybrid-1 and hybrid-2 structures, the second thymine, T18 $\left({ }^{\mathrm{Br}} \mathrm{U}\right)$, of the TTA linker was located quite close to the $\mathrm{C} 1^{\prime}$ of the first deoxythymidine, T17, which can facilitate hydrogen abstraction during photoreaction. Overall, the photoreaction efficiency was determined both by the stacking models of loop bases with G-tetrads and by the TTA loop structure itself. Although in $\mathrm{Na}^{+}$solution the stacking of ${ }^{\mathrm{Br}} \mathrm{U}$, adenine and G-tetrad in the linker region was not as strong as the stacking in $\mathrm{K}^{+}$solution, electron transfer from G-tetrad to the linker loop region may still occur. However, adenines which were not located between ${ }^{\mathrm{Br}} \mathrm{U}$ and G-tetrad could not prevent the electron back transfer and increase the lifetime of the anion radical formed by ${ }^{\mathrm{Br}} \mathrm{U}$.

In conclusion, we have demonstrated that $5^{\prime}$-end FAMlabeled DNA can be used to easily analyze the photoreaction of 5-halouracil-containing DNA via denaturing gel analysis. Using this method, we discovered that the photoreaction of 5-bromouracil-containing DNA could be used to probe the loop structure of G-quadruplexes formed by longer human telomeric DNA. Different photoreaction efficiencies were observed for eight TTAGGG repeats in $\mathrm{K}^{+}$and $\mathrm{Na}^{+}$solutions, indicating different loop structures among two consecutive G-quadruplexes. Dimeric models of the consecutive structures in $\mathrm{K}^{+}$and $\mathrm{Na}^{+}$solutions were also generated by molecular simulation, and the loop regions located between two G-quadruplex units were compared. Therefore, our method can be used to detect higher-order G-quadruplex formation in long human telomeric DNA. Furthermore, as efficient photoreaction was observed in $\mathrm{K}^{+}$solution, which is biologically relevant, this method may be used to probe the G-quadruplex formation of telomeric DNA in cells.

We are sincerely thankful for the JSPS-NSF International Collaborations in Chemistry (ICC) program and the WPI program (WPI-iCeMS, Kyoto University).

\section{Notes and references}

1 W. E. Wright, M. T. Valere, E. H. Kenneth, D. L. Stephen and W. S. Jerry, Genes Dev., 1997, 11, 2801.

2 G. Biffi, D. Tannahill, J. McCafferty and S. Balasubramanian, Nat. Chem., 2013, 5, 182.

3 V. A. Zakian, Exp. Cell Res., 2012, 318, 1456.

4 M. L. Bochman, K. Paeschke and V. A. Zakian, Nat. Rev. Genet., 2012, 13, 770 .

5 Y. Wang and D. J. Patel, Structure, 1993, 15, 263.

6 G. N. Parkinson, M. P. Lee and S. Neidle, Nature, 2002, 417, 876.

7 Y. Xu, Y. Noguchi and H. Sugiyama, Bioorg. Med. Chem., 2006, 14, 5584.

8 K. N. Luu, A. T. Phan, V. Kuryavyi, L. Lacroix and D. J. Patel, J. Am. Chem. Soc., 2006, 128, 9963.

9 A. Ambrus, D. Chen, J. Dai, T. Bialis, R. A. Jones and D. Yang, Nucleic Acids Res., 2006, 34, 2723.

10 H. Q. Yu, D. Miyoshi and N. Sugimoto, J. Am. Chem. Soc., 2006, 128, 15461.

11 Y. Xu, T. Ishizuka, K. Kurabayashi and M. Komiyama, Angew. Chem., Int. Ed., 2009, 48, 833.

12 L. Petraccone, C. Spink, J. O. Trent, N. C. Garbett, C. S. Mekmaysy, C. Giancola and J. B. Chaires, J. Am. Chem. Soc., 2011, 133, 20951.

13 R. Hänsel, F. Löhr, L. Trantirek and V. Dötsch, J. Am. Chem. Soc., 2013, 135, 2816.

14 J. Abraham Punnoose, Y. Cui, D. Koirala, P. M. Yangyuoru, C. Ghimire, P. Shrestha and H. Mao, J. Am. Chem. Soc., 2014, 136, 18062.

15 K. Shinohara, Y. Sannohe, S. Kaieda, K. Tanaka, H. Osuga, Y. Xu, T. Bando and H. Sugiyama, J. Am. Chem. Soc., 2010, 132, 3778.

16 C. Zhao, L. Wu, J. Ren, Y. Xu and X. Qu, J. Am. Chem. Soc., 2013, $135,18786$.

17 L. Petraccone, Top. Curr. Chem., 2013, 330, 23.

18 L. Bauer, K. Tlučková, P. Tóhová and V. Viglaský, Biochemistry, 2011, 50, 7484 .

19 H. Yu, X. Gu, S. Nakano, D. Miyoshi and N. Sugimoto, J. Am. Chem. Soc., 2012, 134, 20060.

20 L. Petraccone, J. O. Trent and J. B. Chaires, J. Am. Chem. Soc., 2008, 130, 16530.

21 T. Oyoshi, A. H.-J. Wang and H. Sugiyama, J. Am. Chem. Soc., 2002, 124, 2086.

22 R. Tashiro and H. Sugiyama, J. Am. Chem. Soc., 2003, 125, 15282.

23 T. Watanabe, R. Tashiro and H. Sugiyama, J. Am. Chem. Soc., 2007, 129, 8163.

24 F. Hashiya, A. Saha, S. Kizaki, Y. Li and H. Sugiyama, Nucleic Acids Res., 2014, 42, 13469.

25 Y. Xu, R. Tashiro and H. Sugiyama, Nat. Protoc., 2007, 2, 78.

26 Y. Xu and H. Sugiyama, J. Am. Chem. Soc., 2004, 126, 6274.

27 R. Tashiro, K. Nakamura and H. Sugiyama, Tetrahedron Lett., 2008, 49, 428.

28 A. Barlev and D. Sen, J. Am. Chem. Soc., 2013, 135, 2596.

29 C. J. Lech, A. T. Phan, M. E. Michel-Beyerle and A. A. Voityuk, J. Phys. Chem. B, 2015, 119, 3697.

30 L. Wu, K. Liu, J. Jie, D. Song and H. Su, J. Am. Chem. Soc., 2015, 137, 259. 